\title{
Detection of Tickborne Relapsing Fever Spirochete, Austin, Texas, USA
}

\author{
Jack D. Bissett, Suzanne Ledet, Aparna Krishnavajhala, Brittany A. Armstrong, Anna Klioueva, \\ Christopher Sexton, Adam Replogle, Martin E. Schriefer, Job E. Lopez
}

In March 2017, a patient became febrile within 4 days after visiting a rustic conference center in Austin, Texas, USA, where Austin Public Health suspected an outbreak of tickborne relapsing fever a month earlier. Evaluation of a patient blood smear and molecular diagnostic assays identified Borrelia turicatae as the causative agent. We could not gain access to the property to collect ticks. Thus, we focused efforts at a nearby public park, $<1$ mile from the suspected exposure site. We trapped Ornithodoros turicata ticks from 2 locations in the park, and laboratory evaluation resulted in cultivation of $3 \mathrm{~B}$. turicatae isolates. Multilocus sequencing of 3 chromosomal loci ( $f l a B, r r s$, and $g y r B$ ) indicated that the isolates were identical to those of $B$. turicatae 91E135 (a tick isolate) and BTE5EL (a human isolate). We identified the endemicity of $O$. turicata ticks and likely emergence of $B$. turicatae in this city.

$\mathrm{G}^{1}$ obally, spirochetes that cause tickborne relapsing fever (TBRF) are neglected pathogens, and diagnosis of this disease is challenging because of its nonspecific manifestations. Signs and symptoms of TBRF include cyclic febrile episodes, nausea, and vomiting (1). The bacterium Borrelia turicatae is the primary known causative species of TBRF in low-elevation, arid regions in the southern United States, and a unique manifestation of this disease is neurologic symptoms that further complicate an accurate diagnosis (2). In Texas, most cases of infection with $B$. turicatae have been associated with cave explorers, outdoor enthusiasts, undocumented migrants, and military personnel $(2,3)$. Currently, it is not mandatory to report a diagnosis of TBRF in Texas.

An additional complication with defining public health effects of infection with $B$. turicatae is the dynamics between the pathogen and its tick vector (Ornithodoros turicata). These ticks have a life span of 10 years and can

Author affiliations: Seton Medical Center, Austin Texas, USA

(J.D. Bissett, S. Ledet); Baylor College of Medicine, Houston,

Texas, USA (A. Krishnavajhala, B.A. Armstrong, J.E. Lopez);

Austin Public Health, Austin (A. Klioueva); Centers for Disease

Control and Prevention, Fort Collins, Colorado, USA (C. Sexton,

A. Replogle, M.E. Schriefer)

DOI: https://doi.org/eid2411.172033 endure starvation for $\geq 5$ years. However, $B$. turicatae remains infectious in these ticks (4). Except for persons bitten in karst formations (topographies formed from dissolution of soluble rocks) that can contain swarms of $O$. turicata ticks (2), attached ticks are rarely seen because these ticks are rapid feeders and transmission of $B$. turicatae occurs within seconds of the tick bite $(5,6)$. In addition, $B$. turicatae is maintained transovarially and tick larvae are difficult to see because of their small size ( 6 ). After feeding, ticks return to their cavity dwelling, which includes wood cracks, leaf litter, and small- and medium-size mammal nests and dens $(2,7,8)$.

Little is known regarding the maintenance of $B . t u$ ricatae in nature. Most laboratory isolates have resulted from feeding field-collected ticks on immunologically naive mice and culturing the spirochetes from infected murine blood (9). B. turicatae has also been cultured from the blood of sick domestic dogs $(9,10)$ and a human (11). Furthermore, there is an absence of $B$. turicatae isolates from wild vertebrates, which further limits understanding the etiology of TBRF.

We report clinical manifestations of TBRF for a patient from Austin, Texas, USA. Using species-specific genetic and antigenic markers (12-14), we identified the etiologic agent as $B$. turicatae. Because access to the alleged exposure site was not available, we collected $O$. turicata ticks in a nearby public park. Collected ticks indicated the endemicity of the vector to Austin. These ticks were evaluated for infection by feeding them on immunologically naive mice. We report the transmission and isolation of TBRF spirochetes in culture medium. Partial sequencing of the flagellin B ( $f l a B), 16 \mathrm{~S}$ rRNA ( $r r s)$, and DNA gyrase $\mathrm{B}($ gyr $B)$ genes (total 2,398 bp) indicated probable emergence of $B$. turicatae in Austin, Texas.

\section{Materials and Methods}

\section{The Patient}

The patient was a 34-year-old previously healthy woman whose illness began on March 29, 2017, when she had a 
headache, myalgias, arthralgias, and malaise. On March 30, 2017, she traveled to California on a previously scheduled trip and was febrile. The patient had a temperature of $104^{\circ} \mathrm{F}$ that increased to $105^{\circ} \mathrm{F}$, at which point she sought medical treatment at an urgent care clinic. Complete blood counts, and levels of electrolytes, blood urine nitrogen, creatinine, and liver enzymes were within references ranges. She was given intravenous fluids, discharged with a diagnosis of a viral illness, and given instructions for symptomatic treatment of this illness.

Over the next 2 days, the patient still had a high fever, which prompted her to return to the urgent care clinic. Given her ongoing signs and symptoms, she was referred to a local hospital emergency department in California where she underwent computed tomographic imaging of her brain and a lumbar puncture for cerebrospinal fluid analysis. Computed tomographic imaging of the brain showed no abnormalities. Analysis of cerebrospinal fluid also failed to demonstrate abnormal findings. It was again concluded that she likely had a viral infection and was discharged from the emergency department with instructions for symptomatic treatment.

On April 2, 2017, she reported a blotchy maculopapular rash that began on her extremities and spread to her trunk. The rash was nonpruritic, persisted for several days, then gradually faded away. The patient returned home to Austin, Texas, with a temperature of $104^{\circ} \mathrm{F}$ and continued to have a mild headache in conjunction with intermittent fever. She did not have nausea, vomiting, or diarrhea. Given her ongoing symptoms, on April 9, 2017 she sought an evaluation at an acute care hospital emergency department. At the emergency department assessment, a hematoxylin and eosin-stained peripheral thin blood smear was prepared for evaluation of bloodborne pathogens.

\section{Real-Time PCR Analysis}

We performed a real-time PCR assay on DNA extracted from the spirochete-positive peripheral thin blood smear. We scraped $10 \%$ of the contents of the slide with a scalpel and placed the contents in a tube containing $200 \mu \mathrm{L}$ of phosphatebuffered saline (GIBCO, Gaithersburg, MD, USA). We then extracted DNA by using a QIACube (QIAGEN, Valencia, CA, USA), a tissue protocol, and an elution of $100 \mu \mathrm{L}$. A total of $5 \mu \mathrm{L}$ of the eluted DNA extract was used per $20-\mu \mathrm{L}$ final volume reactions with primers and probes specific for the B. turicatae glycerophosphodiester phosphodiesterase $(g l p Q)$ gene (forward primer 5'-GCCTGTCAGAATGAAAAA-3', reverse primer $5^{\prime}$-CACCTCTGTGAGCTATAATT-3', and probeFAM-5'-TGAGTATGACAAACAAAAAACCACCA$3^{\prime}$-BHQ) and the $B$. hermsii $g l p Q$ gene (forward primer 5'-TCCTGTCAGGGCGAAAAAAT-3', reverse primer 5'-GCTGGCACCTCTGTGAGCTAT-3', and probe FAM-5'AGTCAAAACCAAAAATCACCA-3'-BHQ). The PCR was performed as described (14). A no template (DNA) sample was used as a negative control, and DNA extracted from $B$. turicatae and $B$. hermsii cultures were used as positive controls.

\section{Immunoblotting}

We also performed immunoblotting for relapsing fever group Borrelia spp. and B. turicatae, as described $(3,13,15)$. We subjected protein lysates from $1 \times 10^{7} B$. turicatae and $1 \mu \mathrm{g}$ of recombinant Borrelia immunogenic protein A (rBipA) to electrophoresis by using Mini PROTEAN TGX Precast Gels (Bio-Rad, Hercules, CA, USA) and transferred them onto Immobilon polyvinylidene difluoride membranes (Millipore, Billerica, MA, USA). rBipA was produced as a thioredoxin fusion protein to facilitate solubility and is $\approx 15 \mathrm{kDa}$ larger than the native protein (13).

We sent a deidentified serum sample collected 50 days after infection to Baylor College of Medicine (Houston, TX, USA) for evaluation. This sample was diluted 1:200 in Tropix I-Block Protein-Based Blocking Reagent (ThermoFisher Scientific, Waltham, MA, USA), and polyvinylidene difluoride membranes were probed for 1 hour. Recombinant protein $\mathrm{G}$ conjugated to horseradish peroxidase (ThermoFisher Scientific) diluted 1:4,000 was used as the secondary molecule, and antibody reactivity was detected by chemiluminescence using the Amersham Enhanced Chemiluminescence ECL Western Blotting Detection Reagent (GE Healthcare, Little Chalfont, UK).

\section{Collection of $\boldsymbol{O}$. turicata Ticks}

Because access to the alleged exposure site was not available, we selected a field site in a public park near the suspected exposure site. We determined that the park was in Austin by using the Jurisdictions Web Map maintained by the Enterprise Geospatial Service Program of the City of Austin (http://www.austintexas.gov/department/gisand-maps). Collection efforts were performed in July and November 2017. We placed $\mathrm{CO}_{2}$ tick traps with dry ice as bait in locations with promising $O$. turicata tick habitats, as described (7). As ticks emerged from leaf litter, we stored them in vials labeled according to collection site and date. We collected 20 nymphal ticks from the first location in July and November 2017. We identified the second location in November 2017 and collected 30 nymphs from this site.

\section{Tick Feedings and Isolation of Spirochetes}

All animal studies were approved by the Institutional Animal Care and Use Committee at Baylor College of Medicine. The laboratory animal program follows standards and guidelines established by the Association for Assessment and Accreditation of Laboratory Animal Care and the National Institutes of Health Office of Laboratory Animal Welfare. Animal husbandry was provided by trained veterinary staff and animal care technicians. 
We randomly selected 10 O. turicata nymphs from each collection vial and allowed them to feed on ICR mice obtained from the Institute of Cancer Research (Philadelphia, PA, USA) (16). Animals were sedated by inhalation of isoflurane (Henry Schein, Melville, NY, USA), and ticks were placed on the shaved abdomen of mice and allowed to feed to repletion. Upon completion of the blood meal, ticks were stored in TTP TubeSpin Bioreactor Tubes (MidSci, St. Louis, MO, USA) and housed at $25^{\circ} \mathrm{C}$ and a relative humidity of $85 \%$.

We examined the 3 mice for spirochete infection for 10 days by nicking the tip of the tail and expressing a drop of blood onto a microscope slide. A coverslip was placed over the blood and examined at $20 \times$ magnification by using a CX33 Trinocular Dark Field Microscope (Olympus, Center Valley, PA, USA). When spirochetes were observed, we obtained a terminal blood sample from the sedated mouse by using cardiac puncture. We centrifuged $\approx 500 \mu \mathrm{L}$ of blood at $5,000 \times \mathrm{g}$ for $10 \mathrm{~min}$ and then inoculated $50 \mu \mathrm{L}$ of serum into $4 \mathrm{~mL}$ of modified Barbour-Stoenner-Kelly (mBSK) medium (17). Cultures were grown at $35^{\circ} \mathrm{C}$ in an atmosphere of $5 \% \mathrm{CO}_{2}$ and examined for spirochetes every 4 days. When exponential growth was reached, we passaged cultures into two 50 -mL culture tubes containing fresh mBSK medium for isolation of genomic DNA and generation of stocks (stored in glycerol). For DNA isolation, we produced spirochete pellets by centrifuging the 50 -mL culture tubes at $8,000 \times g$ for $20 \mathrm{~min}$ and extracted genomic DNA as described (18). We designated the 3 isolates from the mice as BRP1, BRP1a, and BRP2.

\section{Plasmid Analysis and Genetic Typing of Relapsing Fever Spirochete Isolates}

We performed reverse-field electrophoresis to resolve plasmid content, as described (9). In addition to BRP1, BRP1a, and BRP2, we compared genomic DNA plasmid profiles with the 91E135 isolate, which originated in Crockett County, Texas (9). Total DNA was used from the 91E135 isolate passaged 5 times and from the Austin isolates passaged 2 times after initial isolation from mice.

We subjected $500 \mathrm{ng}$ of genomic DNA from each isolate to electrophoresis at $100 \mathrm{~V}$ for $15 \mathrm{~min}$ and at $80 \mathrm{~V}$ for $\approx 40$ hours by using a PPI-200 Programmable Power Inverter (MJ Research, Inc., Waltham, MA, USA). Gels were stained with GelRed Nucleic Acid Stain (Phenix Research Products, Candler, NC, USA) according to the manufacturer's instructions.

We performed multilocus sequencing for relapsing fever Borrelia flaB, rrs, and gyrB genes. Amplicons were generated by using specific primer sets (Table). For the rrs gene, we used primers UniB and FD3 to generate the amplicon, and the remaining internal $r r s$ gene primers were used for sequencing. PCR conditions were an initial incubation at $94^{\circ} \mathrm{C}$ for $2 \mathrm{~min}$, followed by 35 cycles at $94^{\circ} \mathrm{C}$ for $30 \mathrm{~s}$, annealing at $55^{\circ} \mathrm{C}$ for $30 \mathrm{~s}$, and an extension at $72^{\circ} \mathrm{C}$ for $3 \mathrm{~min}$. After the last cycle, an extension was performed at $72^{\circ} \mathrm{C}$ for $5 \mathrm{~min}$. We analyzed PCR products by agarose gel electrophoresis to confirm the expected molecular size. Each amplicon was sequenced by Lone Star Laboratories (Houston, TX, USA) by using specific primers (Table). Chromatograms were analyzed, and poor sequences were trimmed by using Vector NTI software (Life Technologies, Grand Island, NY, USA). We performed a BLAST (https://blast.ncbi.nlm.nih.gov/ Blast.cgi) search with assembled contiguous DNA segments (contigs) to speciate isolates.

\section{Results}

\section{Initial Diagnosis of Relapsing Fever Borreliosis}

The patient was afebrile on initial presentation to the emergency department, but a fever quickly developed (temperature

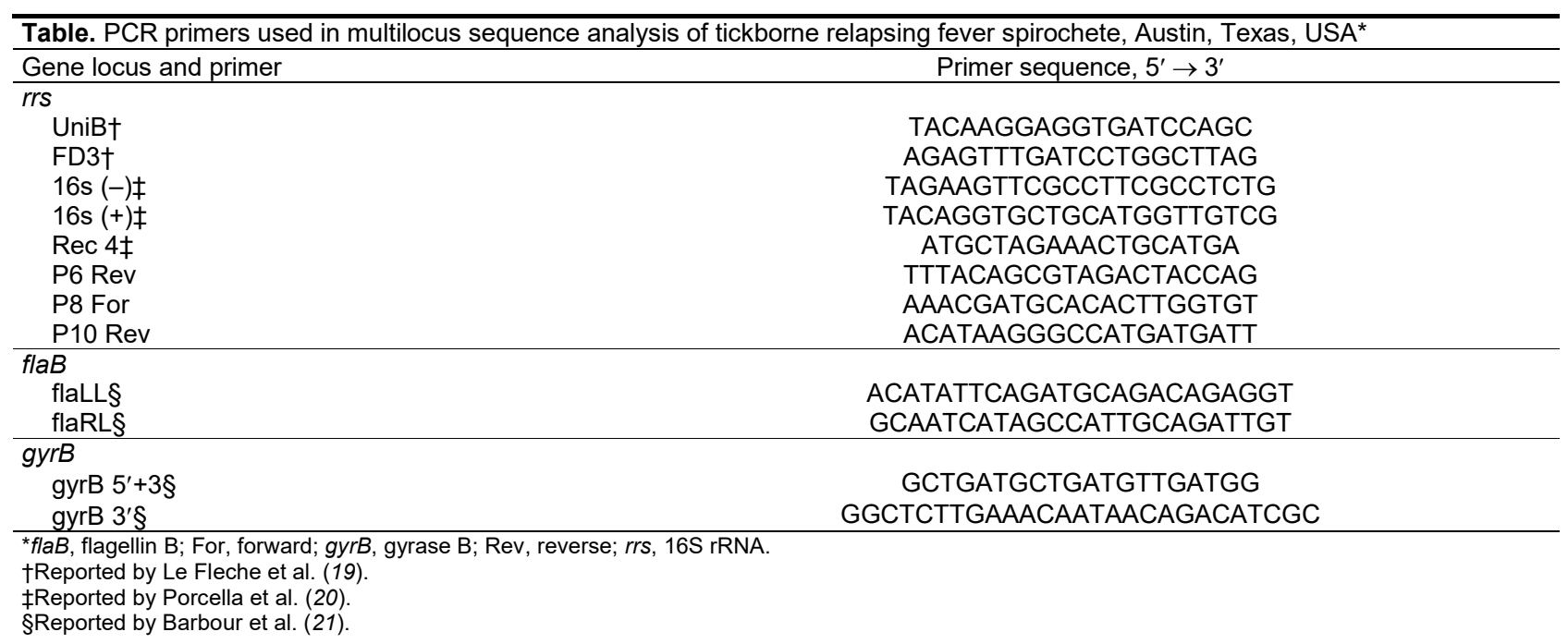


$\left.103.3^{\circ} \mathrm{F}\right)$. Her health deteriorated, and she became tachycardic (heart rate 125 beats/min) and mildly hypotensive (blood pressure 92/38 mm Hg). An initial complete blood count showed a leukocyte count of 6,700 cells $/ \mathrm{mm}^{3}$, a hemoglobin level of $11.1 \mathrm{~g} / \mathrm{dL}$, and a platelet count of $145,000 /$ $\mathrm{mm}^{3}$. Levels of liver enzymes, bilirubin, blood urea nitrogen, and creatinine were within reference limits.

At the hospital visit, the patient reported a travel history to Cancun, Mexico, during February 14-20, 2017, but she had no illness during or upon return from that trip. Also, she had no extensive travel history outside Austin, during February-March 29, 2017, when her illness began. However, during March 24-26, the patient spent a weekend at a rustic conference center in Austin where attendees from throughout the United States converged. Austin Public Health had previously investigated the conference center as the exposure site for an outbreak of TBRF the previous month, but tick trapping efforts were unsuccessful.

During the conference, the patient noticed several insect bites on her legs. However, she did not report seeing ticks. Given her travel history to Cancun, the emergency department physician suspected malaria and requested a peripheral blood smear. Instead of malaria parasites, spirochetes were visualized (Figure 1) and a diagnosis of relapsing fever was made. Treatment with doxycycline was initiated on the evening of hospital admission, and her fever resolved within 24 hours. Modest leukopenia (leukocyte count 3,300 cells $\left./ \mathrm{mm}^{3}\right)$ and thrombocytopenia $(71,000$ platelets $/ \mathrm{mm}^{3}$ ) then developed. Her other signs and symptoms rapidly resolved over the next 3 days, and she was discharged on April 12, 2017.

\section{Diagnosis of Exposure to B. turicatae}

Because blood was not collected during the initial hospitalization of the patient to identify the infectious agent, we retrospectively implemented 2 molecular approaches to identify the causative agent. First, we extracted DNA from a portion of a blood smear and performed a real-time PCR with primers and probe specific for $B$. turicatae $g l p Q$ gene. This assay detected $B$. turicatae but did not detect $B$. hermsii. For this real-time PCR, the amplicon was $67 \mathrm{bp}$, and given the small size, we did not evaluate the sequence or submit it to GenBank.

The average cycle threshold of the assay was 28.81 , and use of primers and probe specific for $B$. hermsii showed negative results. A no template control also showed negative results.

Second, we assessed a serum sample against $B . t u-$ ricatae protein lysates and $\mathrm{rBipA}$. Results also indicated that the patient was infected with B. turicatae (Figure 2). Immunoblotting detected reactivity to 7 protein bands in the $B$. turicatae protein lysate and to rBipA (Figure 2, panel A). A positive control serum sample was used from a

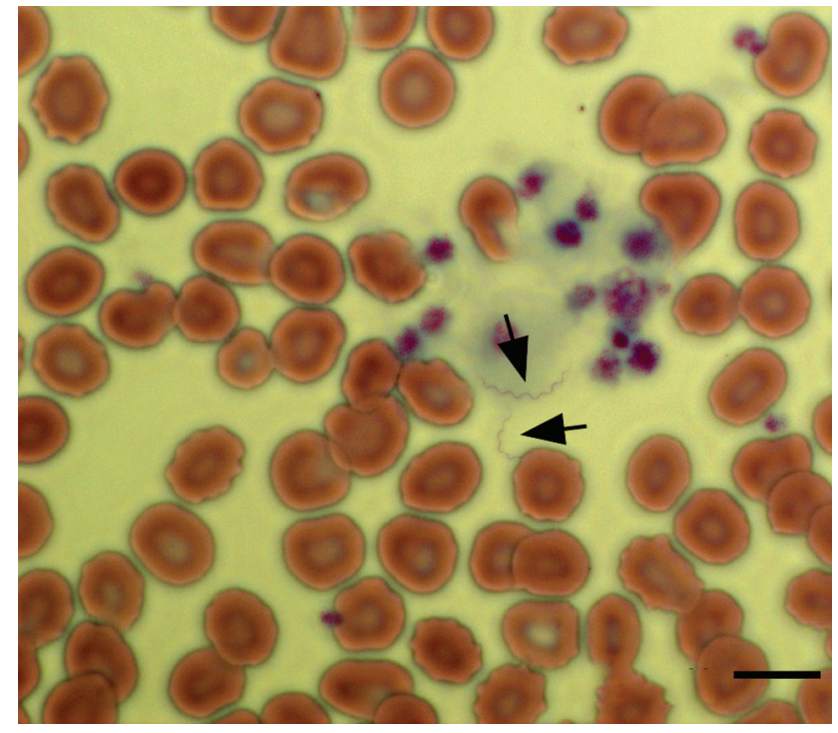

Figure 1. Giemsa-stained peripheral blood smear of a 34-yearold woman with tickborne relapsing fever, Austin, Texas, USA, showing 2 spirochetes (arrows). Scale bar indicates $20 \mu \mathrm{m}$.

previous case report (15), and the negative control human serum sample indicated no serologic cross-reactivity (Figure 2, panels B, C).

\section{Evaluation of Infected O. turicata Ticks from a Public Park}

We collected $O$. turicata ticks in a public park near the rustic conference center from the base of a tree that had 2 dens containing rodent waste, which suggested rodent activity in the area (Figure 3). Ticks emerged from leaf litter $\approx 20$ min after we placed $\mathrm{CO}_{2}$ traps, and we collected ticks $\approx 30 \mathrm{~cm}$ from a cavity opening. Within 5 days after feeding

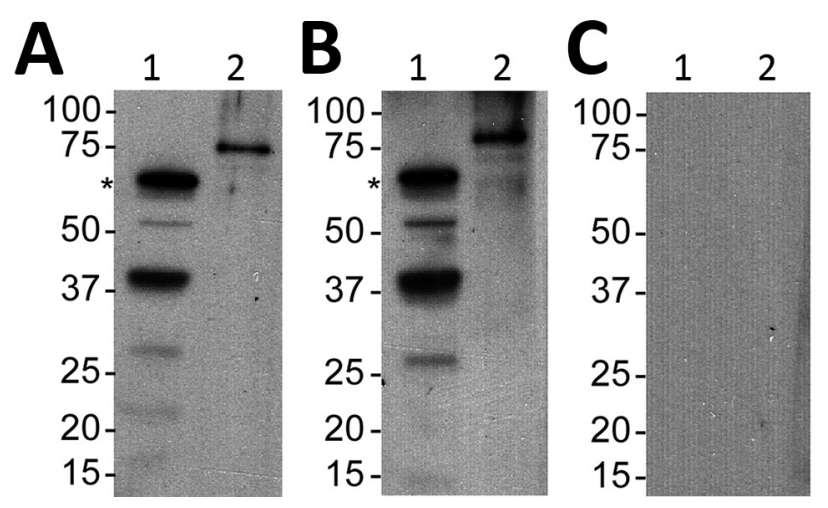

Figure 2. Serologic responses to Borrelia turicatae protein lysates and rBipA in 34-year-old woman with tickborne relapsing fever, detected by immunoblotting, Austin, Texas, USA. A) Serum sample from the case-patient; B) positive control serum sample from another case-patient; C) negative control sample. Lane 1, $B$. turicatae; lane 2, rBipA. Values on the left are in kilobases. Asterisks $\left(^{*}\right)$ indicate the size of native BipA. rBipA, recombinant Borrelia immunogenic protein $\mathrm{A}$. 


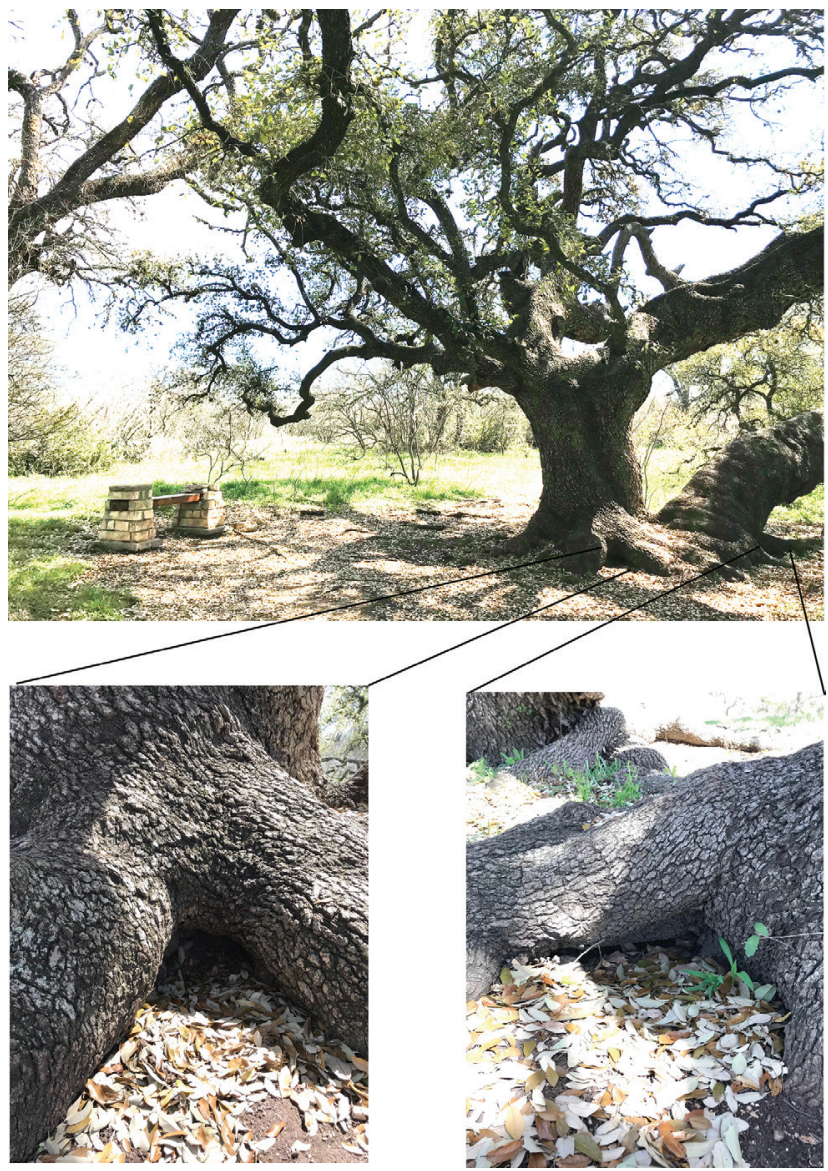

Figure 3. Location of collection sites for Ornithodors turicata ticks, Austin Texas, USA. Two rodent dens (insets) were located at the base of an oak tree. Carbon dioxide traps were placed at the openings until ticks emerged. Borrelia turicatae isolates BRP1 and BRP1a originated from ticks that were collected from the den shown at bottom left, and isolate BRP2 originated from the den shown at the bottom right.

ticks from each location on naive mice, spirochetes were visualized by microscopy in the blood. After we inoculated mBSK medium with a serum sample, spirochetes propagated. Once spirochetes entered the late logarithmic growth phase, we passaged them, which confirmed maintenance of these isolates by vitro cultivation. The 3 isolates were designated BRP1, BRP1a, and BRP2.

\section{Sequence Analysis and Plasmid Diversity of BRP1, BRP1a, and BRP2 Isolates of B. turicatae}

We performed multilocus sequencing to characterize the 3 spirochete isolates that originated in Austin. Sequences of $1,400,566$, and 432 bases were generated for the $\mathrm{AlaB}$, $r r s$, and gyrB genes, respectively (GenBank accession nos. MH503949-51, MH507599-601, and MH507602-04, respectively). Assessment of DNA sequences by BLAST analysis indicated $100 \%$ nucleotide identity to the $91 \mathrm{E} 135$ and BTE5EL isolates of $B$. turicatae. Isolates $91 \mathrm{E} 135$ and BTE5EL originated from field-collected ticks and a febrile soldier from Texas, respectively $(3,9,11)$.

We performed reversed-field gel electrophoresis and identified variation in plasmid diversity between the BRP1, BRP1a, BRP2, and 91E135 isolates of B. turicatae (Figure 4 ). The 3 isolates from Austin contained an $\approx 40-\mathrm{kb}$ linear plasmid that was absent from 91E135. In addition, BRP1 contained an $\approx 60-\mathrm{kb}$ linear plasmid that was absent from 91E135, BRP1a, and BRP2.

\section{Discussion}

We report TBRF in a patient from Austin, Texas. The patient had limited travel history outside Austin in the days before onset of signs and symptoms. Real-time PCR amplification of the $B$. turicatae $\lg Q$ gene and seroconversion for $B$. turicatae $\mathrm{rBipA}$ also helped identify the causative agent. Given that the suspected exposure site was previously investigated by Austin Public Health but ticks were not found, it was useful to collect $O$. turicata ticks near the site and assess their infectious status. Tick transmission feedings resulted in propagation of 3 isolates (BRP1, BRP1a, and BRP2), and results of multilocus sequencing of these isolates were identical to those of a human isolate and a tick isolate $(3,9,11)$. Assessment of total genomic DNA indicated plasmid diversity between the 3 strains and the 91E135 isolate. Ticks that transmitted BRP1 and BRP1a were collected from the same den area at separate times during the year, which suggests circulation of $\geq 2 B$. turicatae variants in the tick population.

Little effort has been invested in active surveillance of TBRF in Texas over the past 70 years $(22,23)$, and more work is needed to understand the burden of this disease and maintenance of the spirochete in nature. Evidence indicates the endemicity of $O$. turicata ticks in San Antonio, Dallas, and Austin $(7,22,24)$, the seventh, ninth, and eleventh most populous cities, respectively, in the United States. $O$. turicata ticks are considered an arthropod reservoir of $B$. turicatae because they have a 10 -year life span and might endure years of starvation while retaining the ability to transmit B. turicatae (25). O. turicata ticks have also been collected in a variety of habitats, including caves, dens, rodent nests, and human dwellings $(2,7,9)$. However, there is a paucity of information regarding mammals involved in maintaining $B$. turicatae in nature.

The life cycle of $B$. turicatae in the vertebrate host consists of periods when the bacterium is undetectable in the blood $(5,13)$. Thus, indirect approaches are needed for improved surveillance. rBipA can support these studies because of the specificity of the protein to TBRF spirochetes $(12,13)$. BipA homologs have not been identified in other pathogens, and previous studies indicated that serologic responses to the protein can discriminate between 


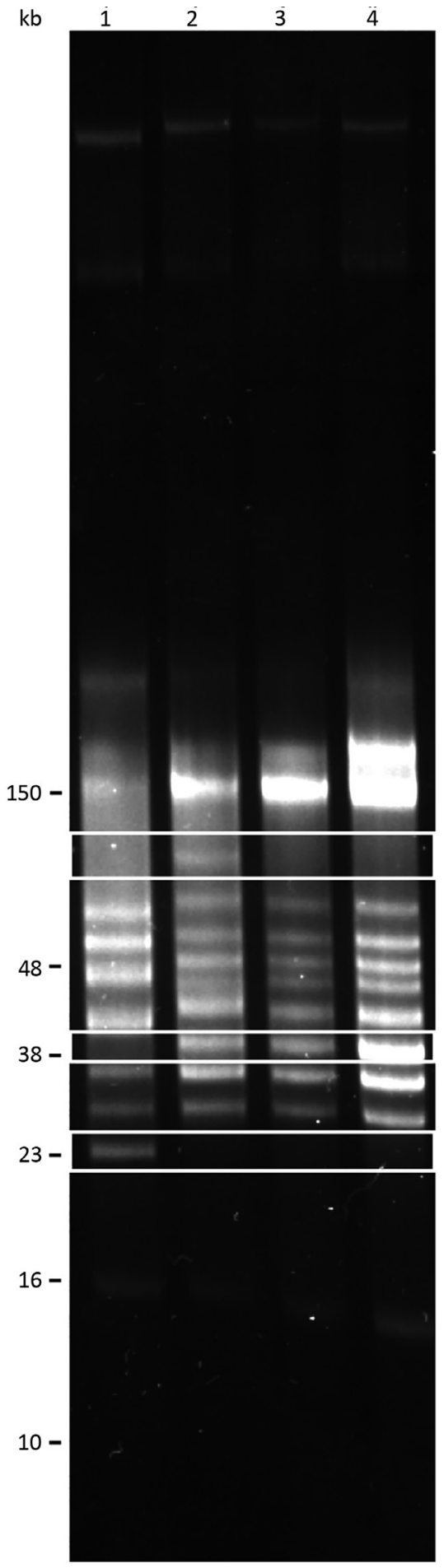

Figure 4. Reversed-field gel electrophoresis of Borrelia turicatae isolates collected from Ornithodors turicata ticks, Austin, Texas, USA (BRP1, BRP1a, and BRP2) and an isolate from previously field-collected ticks (91E135) (9). Lane 1, 91E135; lane 2, BRP1; lane 3, BRP1a; lane 4, BRP2. White boxes indicate a plasmid in BRP1 that is absent from the other strains (top); plasmids unique to BRP1, BRP1a, and BRP2 (middle); and a plasmid in 91E135 strain that is absent in isolates from Austin (bottom). infections caused by Lyme disease and TBRF Borrelia species $(12,13)$. Future efforts will focus on using rBipA as a novel diagnostic antigen for surveillance of $B$. turicatae.

TBRF is typically considered a disease of outdoor enthusiasts and impoverished persons living in primitive conditions $(1-3,11,15,26)$. However, our study suggests emergence of $B$. turicatae in urban areas. The location where ticks were collected was in a densely populated region of the city. However, the maintenance of this pathogen in nature remains unclear. The elusive life cycle of $O$. turicata ticks also poses challenges in understanding the ecology of $B$. turicatae. Furthermore, given the nonspecific clinical manifestations of disease, the public health effect of $B$. turicatae remains vague. Our findings indicate that surveillance efforts should be increased in Austin, Texas, to evaluate emergence of TBRF.

\section{Acknowledgments}

We thank Melissa Solomon for technical contributions to the manuscript.

This study was supported by funds from the National School of Tropical Medicine at Baylor College of Medicine to J.E.L.

\section{About the Author}

Dr. Bissett is an infectious disease physician practicing general infectious diseases in Austin, Texas. His research interests include transplant-related infections, mechanical cardiovascular support device infections, orthopedic device infections, HIV infection, viral hepatitis, and emerging infectious diseases.

\section{References}

1. Dworkin MS, Schwan TG, Anderson DE Jr, Borchardt SM. Tick-borne relapsing fever. Infect Dis Clin North Am. 2008;22:449-68, viii. http://dx.doi.org/10.1016/j.idc.2008.03.006

2. Rawlings JA. An overview of tick-borne relapsing fever with emphasis on outbreaks in Texas. Tex Med. 1995;91:56-9.

3. Christensen AM, Pietralczyk E, Lopez JE, Brooks C, Schriefer ME, Wozniak E, et al. Diagnosis and management of Borrelia turicatae infection in febrile soldier, Texas, USA. Emerg Infect Dis. 2017;23:883-4. http://dx.doi.org/10.3201/eid2305.162069

4. Davis GE. Relapsing fever: the tick Ornithodoros turicata as a spirochetal reservoir. Public Health Rep. 1943;58:839-42. http://dx.doi.org/10.2307/4584474

5. Boyle WK, Wilder HK, Lawrence AM, Lopez JE. Transmission dynamics of Borrelia turicatae from the arthropod vector. PLoS Negl Trop Dis. 2014;8:e2767. http://dx.doi.org/10.1371/ journal.pntd.0002767

6. Davis GE. Ornithodoros turicata:the male; feeding and copulation habits, fertility, span of life, and the transmission of relapsing fever spirochetes. Public Health Rep. 1941;56:1799-802. http://dx.doi.org/10.2307/4583854

7. Donaldson TG, Pèrez de León AA, Li AY, Castro-Arellano I, Wozniak E, Boyle WK, et al. Assessment of the geographic distribution of Ornithodoros turicata (Argasidae): climate variation and host diversity. PLoS Negl Trop Dis. 2016; 10:e0004383. http://dx.doi.org/10.1371/journal.pntd.0004383 
8. Adeyeye OA, Butler JF. Field evaluation of carbon dioxide baits for sampling Ornithodoros turicata (Acari: Argasidae) in gopher tortoise burrows. J Med Entomol. 1991;28:45-8. http://dx.doi.org/10.1093/jmedent/28.1.45

9. Schwan TG, Raffel SJ, Schrumpf ME, Policastro PF, Rawlings JA, Lane RS, et al. Phylogenetic analysis of the spirochetes Borrelia parkeri and Borrelia turicatae and the potential for tick-borne relapsing fever in Florida. J Clin Microbiol. 2005;43:3851-9. http://dx.doi.org/10.1128/JCM.43.8.3851-3859.2005

10. Piccione J, Levine GJ, Duff CA, Kuhlman GM, Scott KD, Esteve-Gassent MD. Tick-borne relapsing fever in dogs. J Vet Intern Med. 2016;30:1222-8. http://dx.doi.org/10.1111/jvim.14363

11. Kingry LC, Batra D, Replogle A, Sexton C, Rowe L, Stermole BM, et al. Chromosome and linear plasmid sequences of a 2015 human isolate of the tick-borne relapsing fever spirochete, Borrelia turicatae. Genome Announc. 2016;4:4. http://dx.doi.org/10.1128/ genomeA.00655-16

12. Lopez JE, Schrumpf ME, Nagarajan V, Raffel SJ, McCoy BN, Schwan TG. A novel surface antigen of relapsing fever spirochetes can discriminate between relapsing fever and Lyme borreliosis. Clin Vaccine Immunol. 2010;17:564-71. http://dx.doi.org/10.1128/ CVI.00518-09

13. Lopez JE, Wilder HK, Boyle W, Drumheller LB, Thornton JA, Willeford B, et al. Sequence analysis and serological responses against Borrelia turicatae BipA, a putative species-specific antigen. PLoS Negl Trop Dis. 2013;7:e2454. http://dx.doi.org/10.1371/ journal.pntd.0002454

14. Breuner NE, Dolan MC, Replogle AJ, Sexton C, Hojgaard A, Boegler KA, et al. Transmission of Borrelia miyamotoi sensu lato relapsing fever group spirochetes in relation to duration of attachment by Ixodes scapularis nymphs. Ticks Tick Borne Dis. 2017;8:677-81. http://dx.doi.org/10.1016/j.ttbdis.2017.03.008

15. Wilder HK, Wozniak E, Huddleston E, Tata SR, Fitzkee NC, Lopez JE. Case report: a retrospective serological analysis indicating human exposure to tick-borne relapsing fever spirochetes in Texas. PLoS Negl Trop Dis. 2015;9:e0003617. http://dx.doi.org/10.1371/journal.pntd.0003617

16. Krajacich BJ, Lopez JE, Raffel SJ, Schwan TG. Vaccination with the variable tick protein of the relapsing fever spirochete Borrelia hermsii protects mice from infection by tick-bite. Parasit Vectors. 2015;8:546. http://dx.doi.org/10.1186/s13071-015-1170-1

17. Battisti JM, Raffel SJ, Schwan TG. A system for site-specific genetic manipulation of the relapsing fever spirochete Borrelia hermsii. Methods Mol Biol. 2008;431:69-84.
18. Simpson WJ, Garon CF, Schwan TG. Analysis of supercoiled circular plasmids in infectious and non-infectious Borrelia burgdorferi. Microb Pathog. 1990;8:109-18. http://dx.doi.org/ 10.1016/0882-4010(90)90075-2

19. Le Fleche A, Postic D, Girardet K, Peter O, Baranton G. Characterization of Borrelia lusitaniae sp. nov. by $16 \mathrm{~S}$ ribosomal DNA sequence analysis. Int J Syst Bacteriol. 1997;47:921-5. http://dx.doi.org/10.1099/00207713-47-4-921

20. Porcella SF, Raffel SJ, Anderson DE Jr, Gilk SD, Bono JL, Schrumpf ME, et al. Variable tick protein in two genomic groups of the relapsing fever spirochete Borrelia hermsii in western North America. Infect Immun. 2005;73:6647-58. http://dx.doi.org/ 10.1128/IAI.73.10.6647-6658.2005

21. Barbour AG, Maupin GO, Teltow GJ, Carter CJ, Piesman J. Identification of an uncultivable Borrelia species in the hard tick Amblyomma americanum: possible agent of a Lyme disease-like illness. J Infect Dis. 1996;173:403-9. http://dx.doi.org/10.1093/ infdis/173.2.403

22. Eads RB, Henderson HE, McGregor T, Irons JV. Relapsing fever in Texas; distribution of laboratory confirmed cases and the arthropod reservoirs. Am J Trop Med Hyg. 1950;30:73-6. http://dx.doi.org/ 10.4269/ajtmh.1950.s1-30.73

23. Brumpt E. Study of Spirochaeta turicatae, n. sp., agent of sporadic recurrent fever of the United States transmitted by Ornithodoros turicata [in French]. Comptes Rendus des Séances de la Société de Biologie et de ses Filiales (Paris). 1933;13:1369.

24. Whitney MS, Schwan TG, Sultemeier KB, McDonald PS, Brillhart MN. Spirochetemia caused by Borrelia turicatae infection in 3 dogs in Texas. Vet Clin Pathol. 2007;36:212-6. http://dx.doi.org/ 10.1111/j.1939-165X.2007.tb00213.x

25. Francis E. Longevity of the tick Ornithodoros turicata and of Spirochaeta recurrentis with this tick. Public Health Rep. 1938;53:2220-41. http://dx.doi.org/10.2307/4582740

26. Dworkin MS, Shoemaker PC, Fritz CL, Dowell ME, Anderson DE Jr. The epidemiology of tick-borne relapsing fever in the United States. Am J Trop Med Hyg. 2002;66:753-8. http://dx.doi.org/10.4269/ajtmh.2002.66.753

Address for correspondence: Job E. Lopez, Department of Molecular Virology and Microbiology and the National School of Tropical Medicine, Baylor College of Medicine, Houston, 1 Baylor Plaza, Houston, Texas 77030, USA; email: job.lopez@bcm.edu 